\title{
PD-L1-Hemmer bei vorbehandelten Patienten mit NSCLC
}

In der BIRCH-Studie wurde die Wirksamkeit des PD-L1-Hemmers Atezolizumab bei Patienten mit fortgeschrittenem nichtkleinzelligem Lungenkarzinom (NSCLC) in der Erst-, Zweit- oder Drittlinie geprüft. Alle Patienten mussten eine PD-L1Expression auf den Tumorzellen bzw. den tumorinfiltrierenden Immunzellen vorweisen.

$\mathrm{n}$ einer großen randomisierten Multicenterstudie bei vorbehandelten Patienten mit NSCLC hatte der PD-L1 („programmed cell death-ligand 1“)-Antikörper Atezolizumab verglichen mit einer Docetaxel-Behandlung zu einem signifikant längeren Überleben geführt [Rittmeyer A et al. Lancet. 2017;389(10066): 255-65]. Besonders hatten dabei Patienten mit nachweisbarer Expression von PD-L1 („programmed cell death-ligand 1“) profitiert. Im Rahmen der vorliegenden Phase-II-Studie BIRCH sollte die Effektivität von Atezolizumab beim NSCLC in Abhängigkeit von der Stärke der PD-L1-Expression und der Zahl der Vortherapien bestimmt werden.
Patienten mit fortgeschrittenem NSCLC konnten in die Studie aufgenommen werden, wenn PD-L1 auf $\geq 5 \%$ der Tumorzellen (TC 2 oder 3 ) oder $\geq 5 \%$ der tumorinfiltrierenden Immunzellen (IC 2 oder 3) exprimiert wurde. Patienten mit Hirnmetastasen, mit vorheriger Pneumonitis und Autoimmunerkrankungen wurden ausgeschlossen. Patienten mit aktivierender EGFR-Mutation oder ALK-Rearrangement mussten entsprechend mit EGFR- bzw. ALK-Inhibitoren vorbehandelt worden sein.

Insgesamt wurden 659 Patienten (median 64 Jahre) in 3 Kohorten mit Atezolizumab (1.200 mg p.i., q3w) behandelt: L1: keine vorherige Chemotherapie $(\mathrm{n}=142) ; \mathrm{L} 2: 1$ vorherige platinhaltige Chemotherapie ( $\mathrm{n}=271)$, und L3: 2 vorherige Chemotherapien, einschließlich einer Platinkombination. In Kohorte L1 wurde die Therapie bis zur Krankheitsprogression fortgesetzt, bei den anderen so lange wie ein klinischer Nutzen feststellbar war. Primäres Studienziel waren die Remissionsraten (ORR) nach RECIST, bestimmt von einem unabhängigen Review-Komitee (IRF). Nach einem Followup von mindestens 12 Monaten betrug

\section{Platinagenzien beim NSCLC im Vergleich}

\section{Carboplatin, Cisplatin und die Frage der Dosis}

Wie wichtig ist beim fortgeschrittenen nichtkleinzelligen Lungenkarzinom (NSCLC) die Wahl des Platinagens und welche Dosis ist bei Einsatz von Cisplatin zu wählen? Antworten liefern die Daten einer aktuellen britischen Studie: Kombiniert mit Gemcitabin war Carboplatin (AUC6; GCb6) Cisplatin in einer Dosierung von $80 \mathrm{mg} / \mathrm{m}^{2}$ plus Gemcitabin (GC80) bzgl. des Überlebens nicht unterlegen, aber toxischer. $50 \mathrm{mg} / \mathrm{m}^{2}$ Cisplatin plus Gemcitabin (GC50) führte zu einem schlechteren Überleben, das nicht durch eine bessere Lebensqualität ausgeglichen wurde [Ferry D et al. Eur J Cancer. 2017;83:302-12]. An der Phase-III-Studie nahmen 1.363 chemotherapienaive Patienten mit NSCLC (Stadium IIIB/IV) teil, die randomisiert GC50, GC80 oder GCb6 erhielten. Primärer Endpunkt war die Überlebenszeit, getestet wurde auf einen Unterschied zwischen den Armen und auf Nichtunterlegenheit (Nichtunterlegenheitsgrenze definiert als Hazard Ratio [HR] von 1,2). Das Überleben unterschied sich signifikant zwischen den 3 Armen $(p=0,046)$ : Im GC50-Arm war es am kürzesten (median 8,2 Monate), unter GC80 lag es bei 9,5 Monaten, unter GCb6 bei 10 Monaten. Die adjustierte HR betrug 1,13 (95\%-Konfidenzintervall [KI] 0,99-1,29) für CG50 versus GC80, und 1,23 (95\%-KI 1,08-1,41) für GC50 versus GCb6. GCb6 war GC80 nicht unterlegen (HR 0,92, obere Grenze des $95 \%$-KI 1,04). Im GCb6-Arm gab es mehr Dosisreduktionen, Therapieverzögerungen und unerwünschte Ereignisse (mind. eine Grad-3/4Toxizität bei $60 \%$ verglichen mit $43 \%$ unter GC80 und $30 \%$ unter GC50).

die ORR $22 \%$, $19 \%$ und $18 \%$ in den Gruppen L1, L2 und L3. Komplettremissionen wurden selten $(\leq 2 \%)$ erzielt. In den Subgruppen mit stärkerer PD-L1-Expression (TC3 oder IC3) war die ORR mit Werten zwischen 31 und $27 \%$ etwas höher. Die Remissionen dauerten im Median etwa 10 Monate, jedoch mit deutlichen Schwankungen abhängig von der Zahl der vorausgehenden Therapien und dem Expressionsgrad von PD-L1. Das progressionsfreie Überleben (PFS) war in der Kohorte L1 am längsten (median 5,4 vs. jeweils 2,8 Monate in L2 und L3). Nach 12 Monaten betrugen die PFS-Raten 20\% (L1), $17 \%$ (L2) und 14\% (L3).

Nach einem Follow-up von 22,5 Monaten war das mediane Überleben aller Patienten der Kohorte L1 mit 23,5 Monaten am günstigsten, gefolgt von L2 und L3 mit 15,5 bzw. 13,2 Monaten. Differenzierte man die L1-Gruppe nach PD-L1-Expression, so hatten die Patienten mit stärkerer PD-L1-Expression (TC3 oder IC3) die beste Prognose mit einem medianen Überleben von 26,9 Monaten. Nach 12 Monaten lebten in der L1-Gruppe noch insgesamt 66,4\% der Patienten, in der L2Gruppe noch 58,1\% und 52,3\% in der L3Gruppe. Dabei war Atezolizumab bei allen Typen des NSCLC wirksam. Auch Patienten mit einer EGFR-Mutation oder einem ALK-Rearrangement sprachen auf den Antikörper an.

Bei 94 \% der Patienten traten Nebenwirkungen auf. Im Vordergrund der Nebenwirkungen standen zahlenmäßig die Fatigue (19\%), Diarrhö (11 \%), Übelkeit (11 \%) und Juckreiz.

Fazit: Die Ergebnisse bestätigen, dass eine Monotherapie mit Atezolizumab den Patienten mit fortgeschrittenem NSCLC einen klinisch relevanten Nutzen bringt. Atezolizumab ist auch bei Patienten mit einer oder mehreren Vortherapien effektiv. Der PD-L1-Status könnte als prädiktiver Biomarker genutzt werden.

Brigitte Schalhorn

Peters S et al. Phase II trial of atezolizumab as first-line or subsequent therapy for patients with programmed death-ligand 1-selected advanced non-small-cell lung cancer (BIRCH). J Clin Oncol. 2017;35(24):2781-9. 